\title{
Perspective
}

PERSPECTIVE Actualité en histoire de l'art

4 | 2006

La monographie d'artiste

\section{Courbet par Clark : visions politiques et visées polémiques d'une monographie}

\section{Thomas Schlesser}

\section{(2) OpenEdition}

1 Journals

\section{Édition électronique}

URL : http://journals.openedition.org/perspective/4228

DOI : 10.4000/perspective.4228

ISSN : 2269-7721

Éditeur

Institut national d'histoire de l'art

\section{Édition imprimée}

Date de publication : 31 décembre 2006

Pagination : 636-641

ISSN : 1777-7852

\section{Référence électronique}

Thomas Schlesser, «Courbet par Clark : visions politiques et visées polémiques d'une monographie », Perspective [En ligne], 4 | 2006, mis en ligne le 31 mars 2018, consulté le 01 octobre 2020. URL : http:// journals.openedition.org/perspective/4228; DOI : https://doi.org/10.4000/perspective.4228 


\title{
Courbet par Clark : visions politiques et visées polémiques d'une monographie
}

\author{
Thomas Schlesser
}

1 Le rôle joué par la monographie de Timothy J. Clark (Image of the People, Gustave Courbet and the 1848 revolution, Londres, 1973 ; trad. fr. : Une image du peuple. Gustave Courbet et la révolution de 1848, Villeurbanne, 1991) sur les questions très classiques de Gustave Courbet et de son esthétique réaliste est incontestablement un des exemples les plus spectaculaires de rupture dans l'historiographie moderne. Elle est le fait d'un historien de l'art anglais, lié dans le courant des années 1960 au mouvement situationniste et à la pensée marxiste. Nous souhaiterions brièvement évoquer les modalités de cette rupture et ses incidences complexes au cours des trente dernières années.

\section{Une histoire de l'art engagée}

Image of the people est issu, comme The Absolute Bourgeois (The Absolute Bourgeois: Artists and Politics in France, 1848-1951, Londres, 1973) de la thèse de doctorat de Clark menée au Courtaud Institute of Art. Les deux ouvrages sont publiés simultanément chez Thames and Hudson, en 1973. Image of the people forme le pan monographique de la thèse, centré sur la première période de la carrière de Courbet, tandis que The Absolute Bourgeois décline différentes conséquences esthétiques de la politique artistique menée sous la Seconde République. Le premier ouvrage constitue une entreprise de redécouverte du peintre franc-comtois à double titre : retrouver Courbet tel qu'en lui-même, au-delà des lieux communs et des légendes émises sur son compte au fil (et spécialement au début) de sa vie par ses contemporains, générer au sujet du peintre une nouvelle méthode d'examen de son engagement et de son œuvre, ne cédant ni à la tendance hagiographique ni à celle du dédain. Penser l'art comme une pratique sociale et saisir l'objet d'art dans ce qu'il a de purement historique et matériel. 
3 Aussi cette monographie est-elle engagée bien au-delà du champ de l'histoire de l'art. Clark travaille sur Gustave Courbet alors qu'une vive agitation intellectuelle rythme la fin des années 1960, et Linda Nochlin, lorsqu'elle fit le compte rendu de l'ouvrage dans Art in America, insiste sur le fait que cette approche développant les liens entre art et histoire, n'aurait pas été possible avant cette période ${ }^{1}$. De son aveu même, Clark a cherché dans la pratique et la perception du maître d'Ornans un éclairage historique sur son propre temps et sur les possibilités d'une révolution sociale. À ce titre, il ne cache pas son amertume lorsque, dans sa préface à l'édition de 1991, il s'interroge sur la lecture de ceux qui furent, vingt ans auparavant, ses alliés français. Les « intellectuels de gauche " n'ont pas tardé, dit-il, à se métamorphoser en "poststructuralistes de l'après Goulag»(p.6). Image of the people est ainsi une monographie militante et l'auteur, en refusant de renier ses lignes, évoque corollairement les engagements bafoués de ces camarades d'antan. Peut-être faut-il rappeler qu'à travers la monographie de Courbet, Clark retraçait : « The real history of the avant-garde [which] is the history of those who by-passed, ignored and rejected it; a history of secret and isolation; a history of escape from the avant-garde and even from Paris itself» (1973, p. 14).

\section{Quelle généalogie?}

4 Pourtant, il y avait déjà eu - avant Image of the people - une monographie militante sur le peintre. En 1952, Aragon avait signé L'exemple de Courbet (Paris, 1952). Mais sa très faible culture politique et sociale du Second Empire rendait ce texte indigent. En occultant ce qui chez Courbet relève du double jeu, de la stratégie, de la connivence, Aragon demeurait prisonnier de l'image de l'artiste brisé, brimé, martyr.

5 Si l'on excepte l'article tout à fait capital de Meyer Schapiro (sur lequel nous reviendrons $)^{2}$, les ouvrages sur Courbet ont toujours fait la part belle à une vision mythologique du peintre: l'autodidacte surclassant l'académisme ambiant, le Communard insoumis, l'amoureux de la vérité, le provincial marchant sur Paris, etc. Certes, le travail de Georges Riat, publié en 1906, est déjà une monographie solide dont la composition tripartite - «les débuts » (jusqu'en 1848), « la gloire » (jusqu'en 1870) et enfin «le déclin » - forme aujourd'hui encore un canon naturel pour appréhender la carrière du maître d'Ornans ${ }^{3}$. Mais, dans la continuité des livres de souvenirs signés Champfleury (Souvenirs et portraits de jeunesse, Paris, 1872), Max Claudet (Souvenirs. Gustave Courbet, Paris, 1878), Émile Gros-Kost (Courbet. Souvenirs intimes, Paris, 1880), Jules Claretie (Courbet, Paris, 1882), Jules-Antoine Castagnary (Gustave Courbet et la colonne Vendôme: plaidoyer pour un ami mort, Tusson, 2000; Exposition des ceuvres de G. Courbet à l'École des beaux-arts (mai 1882), Paris, 1882), Alexandre Schanne (Les souvenirs de Schaunard, Paris, 1892) ou Jules Troubat (Une amitié à la d'Arthez: Champfleury, Courbet, Max Buchon, Paris, 1900), la première moitié du $\mathrm{xx}^{\mathrm{e}}$ siècle a préféré la "figure " monolithique et brossée à grands traits de Courbet - qu'on lise à ce sujet les œuvres de Charles Léger, par exemple (Courbet, Paris, 1934 ; Courbet et son temps, Paris, 1948) - à la place complexe qu'il occupe artistiquement, socialement et politiquement en son temps. L'ensemble des travaux $\mathrm{du} \mathrm{xx}^{\mathrm{e}}$ siècle comme les plus récents reviennent incessamment à ces témoignages de ce que fut et de ce que fit le maître d'Ornans durant sa carrière. Ils les enrichissent - parfois les contestent aussi - avec des éléments historiques plus ou moins importants au fur et à mesure qu'étaient exhumés œuvres, 
archives et articles de presse d'époque. Établie au mitan du $\mathrm{xx}^{\mathrm{e}}$ siècle, l'édition par Pierre Courthion de Courbet raconté par lui-même et par ses amis (Genève, 1948) est symptomatique de ce versant de l'historiographie : il s'agit d'une anthologie de sources premières qui condensait les plus utiles informations sur le chantre du réalisme et offrait simultanément, sous forme fragmentaire, une somme à exploiter.

Clark ne renie pas les efforts et les résultats souvent probants de ses prédécesseurs. Mais il s'éloigne de leur méthode et fonde dans sa monographie une histoire sociale de l'art qui détonne particulièrement en regard d'approches passées trop plates. Surtout, il n'examine plus du tout la qualité et la singularité du réalisme de la même manière. En effet, jusqu'alors, les arguments employés pour défendre la place de Gustave Courbet dans l'histoire de l'art étaient relativement conformes à ceux dont usaient les partisans historiques du peintre : Champfleury, Bruyas, Castagnary, Astruc, Zola... Des toiles du maître étaient ainsi vantées l'énergie de la touche, l'originalité de sujets modernes et la connaissance conjointe des anciens, la monumentalité des formats, la vigueur générale de la composition, des gammes chromatiques : en un mot, la puissance.

Mais Clark puise à d'autres sources et notamment à celle de Meyer Schapiro qui, le premier, a identifié dans le réalisme un usage iconographique de sources populaires. Dans son sillage, Clark a cherché, dans des voies diverses, à comprendre en quoi ce qui apparaissait maladroit ou naïf relevait en réalité d'une complexité esthétique souterraine. Il a ainsi rompu avec l'habituelle réhabilitation de Courbet consistant à en louer la peinture robuste et de tempérament. Non, ce qui intéresse Clark réside précisément dans l'insaisissabilité, l'ambiguïté des représentations sociales et - même le vide d'une esthétique qui, de prime abord, se donne comme foncièrement matérielle. De même, cette insaisissabilité, loin d'être le fruit d'un hasard, d'un instinct mal maitrisé du peintre est, aux yeux de Clark, le produit d'une personnalité fluctuante et très intelligente.

\section{Quelle réception?}

8 La réception de la monographie de Clark en France est intéressante à examiner. Dans la revue Critique d'octobre 1974, Charles Rosen et Henri Zerner proposent un compte rendu à la fois très laudatif et quelque peu méfiant. Ils écrivent ainsi : «Il ne faut pas que les défauts de ces livres [Image of the people et The Absolute Bourgeois] - une pensée parfois imprécise et peu méthodique malgré une recherche de méthodologie, un style un peu journalistique et ronflant à l'occasion - en cachent leurs qualités et leur importance. Il s'agit d'un essai d'histoire sociale et politique de l'art qui cherche, non sans succès, à éviter les pièges habituels du genre. Il ne s'en tient pas à des analogies vagues entre style et idéologie, mais il se refuse à réduire la signification politique d'une œuvre d'art à la réalisation d'un programme explicite. Surtout, il comprend que le rapport de l'idéologie à l'art n'est pas celui d'un contenu à une forme $»^{4}$.

9 Lors d'une reprise partielle de cet article - traduit et adapté par Paul Auster dans le New York Review du 18 mars 1976 - sur les «brilliant provocative volumes " publiés par Clark ${ }^{5}$, Rosen et Zerner insistent : il est indispensable que le public français s'intéresse à cette monographie, la discute, la commente. Eux-mêmes se prêtent au jeu en objectant à Clark certaines lacunes dans son analyse. Reprenant le chapitre sur Un enterrement $\grave{a}$ Ornans, ils pointent au moins trois carences : une incapacité à intégrer les problèmes de «structure et de facture, qui sont pourtant cruciales pour comprendre comment 
Courbet a réussi à représenter ce que les autres ne voulaient pas voir et refusaient d'admettre » (p. 870) ; une esquive du débat quant à la valeur de "l'artifice des moyens de représentation» (p. 871); trop d'imprécision dans l'examen du bouleversement économique généré par les stratégies de Courbet vis-à-vis de son public. Aucun doute possible : cette monographie impressionne Charles Rosen et Henri Zerner qui invitent la communauté scientifique à plancher sur les questions soulevées par le jeune historien anglais.

Ce sera le cas. Chez Linda Nochlin, Klaus Herding, James Henry Rubin ou Patricia Mainardi, on observe un retour et un recours permanent à Clark pour investiguer des questions aussi diverses que les dimensions féministe, démocratique, économique, anarchisante de Courbet et de son œuvre. La question est plus complexe dès lors qu'il s'agit de Michael Fried (Le réalisme de Courbet, Paris, 1993), ce dernier revendiquant une sorte de contre-approche du réalisme. Cependant, cette perspective prend sans cesse en compte celle de Clark, comme une référence face à laquelle il est indispensable de se situer ${ }^{6}$.

Quoi qu'il en soit, le vœu émis dans l'édition française de Image of the people, en 1991 (qu'elle soit « un ensemble de paramètres formant le cadre le plus propice à un travail ultérieur de description et d'analyse »,1991, p. 8) a d'abord été efficace auprès de la recherche anglophone. La monographie de l'écrivain australien Jack Lindsay s'inscrit de manière explicite ${ }^{7}$ dans sa continuité en poursuivant des investigations de nature similaire. Cela ne signifie pas que tous les courbetiens anglophones souscrivent aveuglément à la démarche de Clark. Il suffit de lire l'intéressant article d'Alan Bowness justement intitulée « The New Courbet Literature » dans The Burlington Magazine d'avril 1977 pour le constater ${ }^{8}$. La grille de lecture politique de l'auteur est perçue comme abusive voire déformante. Bowness, affirmant ouvertement sa préférence pour la monographie de Lindsay, regrette la part d'artifice de celle de Clark : les contemporains de l'artiste, à ses yeux, ressemblent à des figures de théâtre sur une scène. L'attaque est sévère.

Mais malgré ce type de critiques, Clark a grandement inspiré certains chercheurs dans leur démarche. On doit notamment signaler, dix ans après l'article de Bowness, l'exposition Courbet reconsidered ${ }^{9}$, dans laquelle Linda Nochlin, Sarah Faunce et Patricia Mainardi, héritière des avancées de Clark, oseront livrer au grand public des approches audacieuses, et parfois peut-être excessives ${ }^{10}$, du contenu esthétique du réalisme du maître d'Ornans.

\section{Clark en France aujourd'hui}

Sans conteste, Image of the people a servi de base à l'indispensable monographie d'Un enterrement à Ornans par Jean-Luc Mayaud (Courbet, l'Enterrement à Ornans : un tombeau pour la République, Paris, 1999). Historien avisé du monde rural et de la Franche-Comté, l'auteur prolonge les travaux de Clark en examinant avec une extrême précision l'ensemble des subtilités idéologiques véhiculées par l'identité des protagonistes de la toile et en approfondissant les problèmes de sa réception. Il montre comment, dans Un enterrement à Ornans, la singularité de chacun des parcours politiques des personnages ${ }^{11}$ entre en tension avec l'impression première de masse compacte, vile et sale d'un monde paysan et bourgeois auquel est accordée la noblesse d'une peinture d'histoire. "Ce sont plusieurs tendances du républicanisme qui sont incarnées par les figurants de 
l'Enterrement» (p. 49), explique Mayaud qui ajoute : «Force est de constater que le tableau a été manipulé » (ibid.) Et c'est la signification des manipulations doublée d'une nouvelle lecture - ajustée aux découvertes archivistiques de l'historien - qui (r)établissent, comme Clark le souhaitait, la profonde complexité idéologique du réalisme de Courbet. Bertrand Tillier procède de même dans ses études des rapports entre Courbet et le champ politique ${ }^{12}$. Il soulève les problèmes de la réception de l'artiste chez les caricaturistes, l'évolution de la perception de son œuvre pendant et après la Commune. Pas plus que Mayaud, il ne revendique une quelconque filiation et encore moins une appartenance à une école de pensée, mais il répond à sa manière à certaines des nombreuses questions que pose la monographie de Clark. De telles entreprises sont salutaires et unanimement reconnues comme telles. Il n'empêche : parmi la prolifération de monographies sur le peintre, on peine franchement à en trouver qui prennent en compte celle de Clark. Non qu'elles l'ignorent, loin s'en faut ! Mais elles en tarissent la portée en se limitant à des citations pleines de déférence plutôt qu'en s'engouffrant dans des brèches ouvertes pourtant de longue date.

On pourrait, à peu de choses près, faire le même constat à propos de Michael Fried. En bénéficiant d'une traduction chez Gallimard en 1993, Courbet's Realism a très vite été une référence incontournable sur Courbet, mais il ne pouvait être discuté de la même manière qu'Image of the People. Parce qu'il est à la fois ouvertement spéculatif (l'auteur glosant sur les intentions inconscientes du peintre) et parfaitement clos sur lui-même, Courbet's Realism n'ouvre pas de champ de réflexion : il l'occupe entièrement. Son parti pris ne peut guère susciter qu'un rejet en bloc ou qu'un vif intérêt (le plus souvent d'ailleurs), mais il n'offre pas de friche à exploiter. Chez Clark, le souci est tout différent: la lucarne qu'il ouvrait ne devait en aucun cas servir de point final aux recherches sociopolitiques sur le peintre. Malheureusement, ce fut plutôt le cas. Louer et citer Image of the people pour ne pas s'aventurer davantage dans des réflexions personnelles sur les enjeux idéologiques entourant Courbet, son œuvre et sa perception semble un exercice convenu et passé dans les mœurs.

15 L'exposition orchestrée par Hélène Toussaint au Grand Palais ${ }^{13}$ esquive trop souvent les enjeux sociopolitiques. Qu'on lise à ce sujet la notice d'Une après-dînée à Ornans par exemple : il apparaît clairement que c'est le rattachement à une tradition "réaliste " (Le Caravage) qui intéresse la commissaire ${ }^{14}$. Reconnaissons que les enjeux politiques ne sont cependant pas totalement absents: en décryptant, sous l'angle de la francmaçonnerie, la charade qu'est L'atelier, le catalogue favorisait l'appréhension de l'œuvre de Courbet comme art à double fond, ambigu, indéterminé.

Prenons à présent deux exemples de monographies : le Courbet de Bruno Foucart sorti en 1977 et réédité en 1995, tout d'abord. Foucart reprend un ouvrage sorti lors de l'exposition au Grand Palais sans en modifier le contenu, dans la collection " monographie» de l'éditeur ${ }^{15}$. Il ouvre son livre sur un chapitre qui fait curieusement écho à celui qui lance Image of the people. Il l'intitule «L'homme et le mythe » là où Clark parlait de "The Courbet legend». Mais, tout en citant la qualité des travaux de Clark, tout en louant en plusieurs occurrences le rôle essentiel de sa monographie dans la compréhension de Courbet, Foucart reprend à son compte la complexité du maître d'Ornans pour insister précisément sur les aspects les plus éloignés des dimensions sociopolitiques de sa peinture. Soixante pages de sa monographie sont ainsi consacrées aux «lyrismes» (chap. 4) de l'artiste. Puisque Clark avait débusqué une complexité cachée chez Courbet, il devenait possible de multiplier les points de vue sur le peintre 
et, sous couvert de cette complexité, se présentait l'opportunité de revenir très exactement à ce que Clark souhaitait dissiper. Certaines envolées baudelairiennes de B. Foucart attestent amplement cette dérive: "Cet extraverti, ce sanguin, grand raconteur d'histoires énormes et sonores, cet agitateur d'idées révolutionnaires est en fait dans son intime le poète de la paix, du silence, de ce paradis perdu des origines où tout est calme, simplicité et dormition" (p.36). D'une édition l'autre, chez le même prestigieux éditeur, tout est donc assumé de la même manière à dix-huit ans d'intervalle: l'auteur se félicitait pourtant qu'enfin Courbet fût restitué comme individu « ondoyant et divers » (p. 9). Était-ce grâce à la monographie de Clark? Non : B. Foucart l'affirme clairement : c'était grâce aux travaux d'André Fermigier ${ }^{16}$. Ceux-là qui avaient montré que "Courbet est d'abord un peintre sensuel, lyrique, qu'un bouquet de fleurs émeut aussi fort que les spectacles des misères du grand chemin » (p. 8).

Dans un registre assez similaire, la monographie de Valérie Bajou (Gustave Courbet, Paris, 2003) donne une étrange impression. De longs extraits des ouvrages de Clark sont employés pour éclairer des œuvres comme Les casseurs de pierres ou Les paysans de Flagey par exemple. Mais voilà qui semble un recours automatique et presque facile, éludant tout prolongement et toute discussion. Comme Clark le craignait, son décryptage des " tableaux de Courbet à travers les réactions de son premier public (ce qu['il] considère toujours comme essentiel [en 1991]) [...] finit trop souvent par vouloir passer pour le compte rendu des tableaux en question » (p. 8 de l'édition française). Image of the people devient dès lors une caution toute faite. L'auteur s'en remet à son contenu, l'évacue en vitesse et préfère multiplier des descriptions confinant souvent à la sensiblerie. L'« effet hypnotique " propre au travail de la monographie, selon V. Bajou (p. 398), procure le sentiment que celui-ci relève parfois du prétexte, dans une approche parfois trop personnalisée.

Le travail de Clark semble aujourd'hui cité - en France du moins - comme une espèce d'étape historique et comme une sage référence, alors qu'il est, au même titre que celui de Michael Fried, un moteur à investigations. Sans doute une traduction homogène du triptyque que forment Image of the people, The Absolute Bourgeois et The Painting of Modern Life $^{17}$ sera-t-elle salutaire pour redonner le goût d'une histoire sociale de l'art, dont on dira trop vite qu'elle est « datée » alors qu'elle n'est en rien épuisée. On peut louer les efforts d'Art édition qui, en 1991, entreprit de publier en français ces trois titres capitaux. Cependant, la qualité relative des traductions des deux premiers opus associée à l'absence de traduction du troisième ${ }^{18}$, rend ce louable effort un peu vain. Le projet d'une publication française et intégrale des trois volumes à l'occasion de la prochaine exposition Courbet par les Presses du réel permettra assurément de montrer que loin d'être un mythe figé dans l'historiographie, le travail de Clark est une approche aussi vivante et stimulante de l'histoire de l'art que l'était l'art de Courbet vis-à-vis de l'histoire. 


\section{NOTES}

1. Linda Nochlin explique : "Clark's approach to mid-19th century art history is a self-conscious and highly sophisticated one; it is also a way of thinking about both art and history that would not have been possible before the 1960's : that is to say, it is conditioned not only by the political events and experiences of the times, but, no less importantly, by the alternatives to conventional empirical, positivistic and naïvely 'neutral history', newly avalaible through the innovations of French structuralists like Barthes, Lacan, Goldman and Foucault in a wide range of disciplines ", dans Art in America, septembre-octobre 1974, p. 51.

2. Meyer Schapiro, "Courbet and popular imagery: An essay on realism and Naiveté », dans Journal of the Warburg and Courtauld Institute, $\mathrm{n}^{\circ} 4$ (1941), p. 164-191; trad. française dans Style, artiste et société, Paris, 1982.

3. Georges Riat (Gustave Courbet peintre, Paris, 1906) se livre à un travail minutieux, axé sur le climat polémique qui entoure le peintre. Ce dernier n'est jamais définitivement rangé sous une bannière politique ou artistique par le biographe qui montre au contraire comment Courbet et son œuvre entrent dans de nombreuses logiques, parfois contradictoires, notamment auprès des autorités du Second Empire.

4. Charles Rosen, Henri Zerner, «L'antichambre du Louvre ou l'idéologie du fini », dans Critique, n³29, octobre 1974, p. 869.

5. Charles Rosen, Henri Zerner, «The Revival of Official Art ", dans New York Review, 18 mars 1976, p. 34.

6. Sur cette comparaison entre l'approche de Clark et celle de Fried, on renverra volontiers le lecteur à Terry Atkinson, «Beholding Courbet from the Side », dans Oxford Art Journal, 15/1, 1992, p. 108.

7. Jack Lindsay écrit : «Important interpretations, bringing out the links with popular art, have however been made by Schapiro and Nochlin. Here was the first break-through to a fresh perception of Courbet's aims and achievements; and a serious attempt to discard the old superficial judgments and see the real social bases of art has been made by Clark ", dans Gustave Courbet, His Life and Art, New York, 1973, p.X.

8. Alan Bowness, "The New Courbet Literature », dans The Burlington Magazine, CXIX, n' 889 , avril 1977, p. 290-291.

9. Sarah Faunce, Linda Nochlin éd., Courbet reconsidered, (cat. expo., New York, Brooklyn Museum, 1988-1989), New Haven, 1988.

10. C'est là un élément important qui mériterait de multiples développements : à compter de Clark, et plus encore de Fried, les problèmes de la «sur-interprétation » de l'œuvre de Courbet sont récurrents. Ils ont fait l'objet d'une étude et d'une communication de Ségolène Le Men lors du congrès «L'esthétique en actes » (congrès, 2005, Paris). On lira aussi sur le cas de L'atelier la thèse de Frédérique Desbuissons : Énigme et interprétations : L'atelier du peintre de Gustave Courbet, université Paris I, 1997.

11. Sont présents la génération des républicains de l'an II (les pères Secrétan et Cardey au centre, le grand-père Oudot à droite), le monde judiciaire au service de l'État (Hyppolite Proudhon au centre), la bourgeoisie libérale (Prosper Teste au centre), la génération des quarante-huitards confinant au socialisme radical (Buchon, tout au fond du cortège, le porteur Promayet, Sage derrière les bedeaux).

12. De Bertrand Tillier, on consultera entre autres: "Sur quelques caricatures vaches contre Gustave Courbet ", dans Collectif, L'Animal en politique, Paris, 2003 ; « Le peintre déboulonné par la caricature ", dans Courbet et la Commune, (cat. Expo., Paris, Musée d'Orsay, 2000), Paris, 2000 ; Jules-Antoine Castagnary, Gustave Courbet, Bertrand Tillier éd., Rezé, 2000 ; Jules-Antoine 
Castagnary, Gustave Courbet et la colonne Vendôme : plaidoyer pour un ami mort, Bertrand Tillier éd., Tusson, 2000.

13. Hélène Toussaint éd., Gustave Courbet, (cat. expo., Paris, Galeries nationales du Grand Palais, 1977-1978), Paris, 1977.

14. Au sujet d'Une après-dînée, Hélène Toussaint explique qu'il « est impossible que Courbet n'ait pas vu une gravure [de la Vocation de Saint Matthieu] ou qu'un Romain ne lui en ait rapporté un dessin, voire une copie », dans Ibid., p. 96.

15. La collection est ainsi présentée: «Les grands écrits monographiques irremplaçables par l'apport de leur texte à la connaissance de l'artiste ».

16. André Fermigier, Courbet, Genève, 1971.

17. Timothy J. Clark, The Painting of Modern Life : Paris in the Art of Manet and his Followers, Londres, 1985.

18. Ouvrage qui souffrit d'une réception française pour le moins mitigée. Françoise Cachin s'employa à l'éreinter violemment dans le New York Review of Books du 30 mai 1985. S'ensuivit un jeu classique de droits de réponse. Le prétexte était attendu : l'ouvrage cédait à une idéologie qui - sans doute - n'était pas celle de Françoise Cachin: "Clark himself, formerly at Leeds University, writes in this book like a new breed of pilgrim who has come to the shores of Hawthorne's New England seeking to breathe new life into a dying Puritanism with the formulas of historical materialism ».

\section{INDEX}

Keywords : art, history, social art history, militant monograph, myth

Mots-clés : art, histoire, histoire sociale de l'art, monographie militante, mythologie

Index chronologique : 1900

\section{AUTEURS}

\section{THOMAS SCHLESSER}

Université Paris X - Nanterre 